\title{
A NEW DEBLOCKING ALGORITHM BASED ON ADJUSTED CONTOURLET TRANSFORM
}

\author{
Haohao Song, Songyu Yu, Chen Wang, Li Song, Hongkai Xiong \\ Institute of Image Communication and Information Processing \\ Shanghai Jiao Tong University, Shanghai, China
}

\begin{abstract}
A new postprocessing method based on adjusted contourlet transform is introduced in this paper for suppressing blocking artifacts (BA) in block-based discrete cosine transform (BDCT) compressed images. To our best knowledge, this is the first time contourlet is applied to this field. By exploiting scale space edge detector (ss-edge detector), our algorithm can extract and protect blocking map (BM) and edge map (EM) in the compressed image respectively in the same time. By transforming the compressed image into adjusted contourlet domain, the adaptive thresholds are obtained according to BM. According to the adaptive thresholds, the contourlet coefficients in different subbands are filtered. Experimental results show that our deblocking algorithm achieves better performance than the other iterative and noniterative methods reported in the literature.
\end{abstract}

\section{INTRODUCTION}

In the past decade, many postprocessing methods have been proposed for suppressing BA to improve the visual quality of BDCT compressed images. Liu and Bovik proposed a blind measurement and reduction of BA in DCT domain [1]. Some deblocking methods using wavelet representation are proposed $[2,3]$. But compactly supported wavelets can lead to many visual artifacts when used in conjunction with nonlinear processing, particularly for decimated wavelet used at critical sampling. In [4], noniterative, spatially adaptive postfiltering is proposed for image deblocking. However, at low bit rates, block discontinuities cannot be completely eliminated by spatially adaptive postfiltering. The projection onto convex sets (POCS) algorithms $[5,6]$ deblock images by iterating the projections onto the quantization constraint set and the image smoothness constraint set until convergence. A major concern with the POCS method is the computational complexity, although it can usually produce good results. Recently, image deblocking algorithms using overcomplete wavelet representation are proposed $[7,8]$. The overcomplete wavelet representation enables a multiscale edge analysis and is useful for edge-based image processing.

Contourlet $[9,10]$ is presented by Do and Vetterli as a new image representation method. It can efficiently represent image containing contours and textures. Contourlet-based denoising methods have shown great potential and are very competitive with wavelet-based denoising methods $[11,12]$. Random noise will generate significant wavelet coefficients just like true edges, but is less likely to generate significant contourlet coefficients [10] Because the values of coefficients corresponding noise are very low, it is very easy to remove noise by using thresholding method. It is the advantage offered by the contourlet transform. Based on the advantage of contourlet and the good results in the domain of image denoising above, we propose a new deblocking algorithm based on contourlet transform in this paper.

\section{SCALE SPACE EDGE DETECTOR AND ADJUSTED CONTOURLET}

Ss-edge detector can locate and characterize scale space edges in the luminosity of an image. Its edge definition, developed by Lindeberg, adaptively selects appropriate scale(s) at each point in the image. The sparse scale space search and edge following algorithm is designed to flexibly limit computational resource needs and preserve edge connectivity [13]. Ss-edges implementation iteratively climbs the scale space gradient until an edge point is located and then iteratively steps, perpendicular the gradient, along a segment, repeating the gradient climb, to fully extract an ordered edge segment. The advantage of ss-edge detector, compared to a global search method, is in its use of computational resources, flexibility of choosing the search space, and flexibility of specifying the details of its edge finding/following. Fig. 1 shows the edge detecting result of the compressed "Lena" image (Quantization tables Q3 in [8] are used) by ss-edge detector. It is evident that ss-edge detector can extract object edges and BA in the same time exactly, and their strengths are different. We can classify object edges and BA according to their strength in detecting result to obtain EM and BM, which are the basis of our algorithm. 


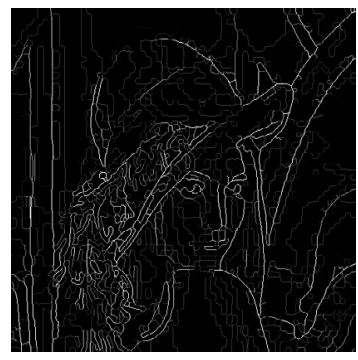

Fig. 1. Edge detecting result of the compressed "Lena" image.

Contourlet was proposed by Do and Vetterli at 2001. It is a double filter bank structure for obtaining sparse expansions for typical images with smooth contours. In this double filter bank, the Laplacian pyramid (LP) is first used to capture the point discontinuities, then followed by a directional filter bank (DFB) to link point discontinuities into linear structures. The overall result is an image expansion using basic elements like contour segments, and thus is named contourlet [10].

It is desired that there is no downsampling operation in the deblocking algorithm, which can make the position of the block boundaries in each scale consistent with those in the spatial domain and facilitate the removal of block discontinuities at those positions. However, traditional contourlet transform (CT) includes downsampling, which can make block boundaries and edges departure from original positions. To overcome this defect of traditional CT, we propose an adjusted CT (ACT), and use it in our deblocking algorithm. The flowchart of ACT is shown as Fig. 2.

For traditional CT, high-frequency (HF) subbands are decomposed in the column direction as soon as are decomposed in the row direction and downsampled. Different from traditional CT, downsampled rowdecomposing subbands are reconstructed immediately in our ACT. The scheme can ensure that the following columndecomposing subbands have the same size as original subbands. Consequently, we can find the position of the block boundaries and edges in each scale exactly based on BM and EM.

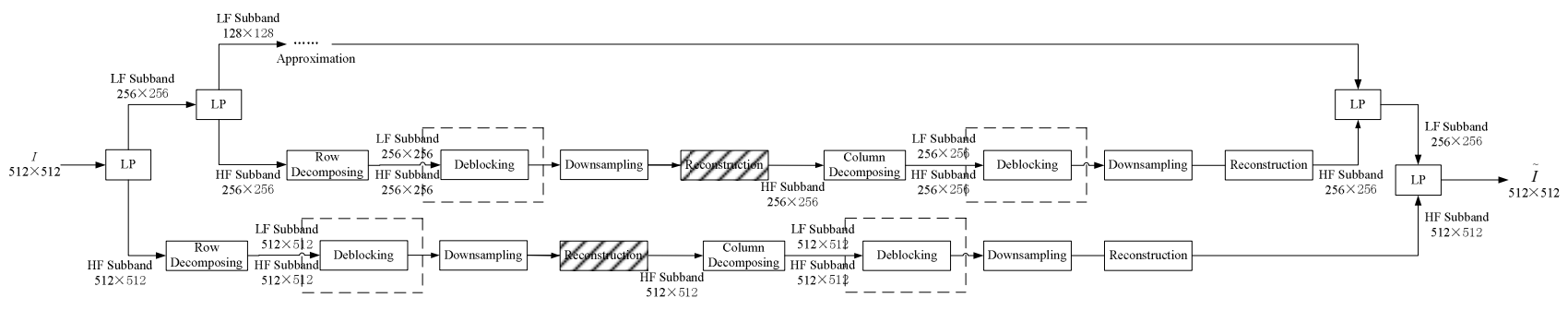

Fig. 2. Flowchart of ACT.

\section{THE PROPOSED DEBLOCKING ALGORITHM}

Our algorithm is implemented in the position as the dashed frames in Fig. 2.

\subsection{Extraction of $B M$ and $E M$}

The first step in our algorithm involves extraction of BM and EM. By exploiting ss-edge detector, object edges and $\mathrm{BA}$ can be extracted in the same time. It is more important that they have the different strengths in detecting results (The strength of object edges is higher than that of BA evidently). We classify them based on their strengths formulated as eqn. (1), and obtain corresponding EM and BM. Fig. 3 shows the classification results of Fig. 1. Here, $V(i, j)$ is the value of $(i, j)$, Thres $_{\text {edge }}=128$, Thres $_{\text {block }}=5$. We assume that pixels whose values are less than Thres $_{\text {block }}$ have no effect for visual sense and do not cause BA.

$$
\left\{\begin{array}{lc}
E I(i, j)=1 & V(i, j) \geq \text { Thres }_{\text {edge }} \\
B M(i, j)=1 & \text { Thres }_{\text {block }} \leq V(i, j)<\text { Thres }_{\text {edge }}
\end{array}\right.
$$

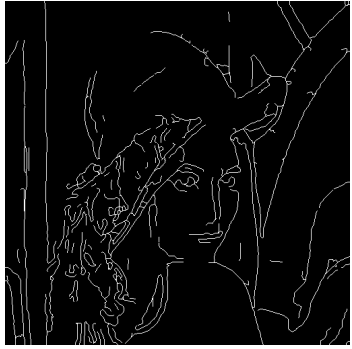

(a)

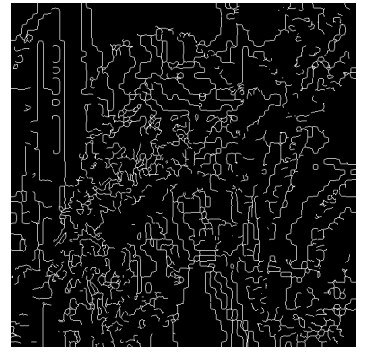

(b)
Fig. 3. Classification results of Fig. 1 (a) EM (b) BM.

According to BM and EM that are obtained by ss-edge detector in the same time, we filter block coefficients and edge coefficients respectively in different subbands with adaptive thresholds. Therefore, our deblocking algorithm can protect object edges efficiently when deblocking the compressed images.

\subsection{ACT and Evaluation of Adaptive Thresholds}

In our algorithm, the compressed image is transformed by 3scale adjusted contourlet representation. The filters should 
be short to keep the computational burden low and avoid smearing image details, therefore, the $5 / 3$ biorthogonal filters is used for LP and the directional decomposition.

As evaluating the thresholds of the first scale subbands, $\mathrm{BM}$ is used to orient the positions of BA. The threshold for each decomposed subband in the first scale can be calculated based on eqn. (2):

$$
\begin{aligned}
& \text { Thres }_{1 p}=\sum_{V(i, j) \geq 0} V(i, j) / N_{p} \\
& \text { Thres }_{1 n}=\sum_{V(i, j)<0} V(i, j) / N_{n} \quad \text { if } B M(i, j)=1
\end{aligned}
$$

Here, Thres $_{1 p}$ is positive threshold, and Thres ${ }_{1 n}$ is negative threshold in the first scale; $N_{p}$ is the number of positive coefficients, and $N_{n}$ is the number of negative coefficients in corresponding subband.

It is necessary to notice that there are four subbands in the first scale (The other scales are same as it): two subbands after row decomposing and two subbands after column decomposing (both before downsampling). Their sizes are same as original image. Therefore, the orientation of BA is very exact in our algorithm.

Moreover, we adopt the different thresholds to filter BA and edges. The scheme can protect object edges not be smoothed excessively when suppressing BA. Thereby, there are four adaptive thresholds for each subband: Thres $_{1 b p}$ (Positive threshold for BA), Thres ${ }_{1 e p}$ ( Positive

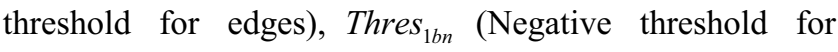
BA), Thres ${ }_{1 e n}$ (Negative threshold for edges). They are calculated based on eqn. (3).

$$
\begin{aligned}
\text { Thres }_{1 b p} & =C_{1 b} \text { Thres }_{1 p}, \text { Thres }_{1 b n}=C_{1 b} \text { Thres }_{1 n} \\
\text { Thres }_{1 e p} & =C_{1 e} \text { Thres }_{1 p}, \text { Thres }_{1 e n}=C_{1 e} \text { Thres }_{1 n}
\end{aligned}
$$

We can get the thresholds corresponding to subbands of coarser scales approximately by the following eqn. (4).

$$
\begin{aligned}
& \text { Thres }_{2 b p}=C_{2 b} \text { Thres }_{1 p} \text {, Thres } \text { Thn }_{2 b}=C_{2 b} \text { Thres }_{1 n} \\
& \text { Thres }_{2 e p}=C_{2 e} \text { Thres }_{1 p} \text {, Thres }{ }_{2 e n}=C_{2 e} \text { Thres }_{1 n} \\
& \text { Thres }_{3 b p}=C_{3 b} \text { Thres }_{1 p} \text {, Thres } 3 b n=C_{3 b} \text { Thres }_{1 n} \\
& \text { Thres }_{3 e p}=C_{3 e} \text { Thres }_{1 p} \text {, Thres }{ }_{3 e n}=C_{3 e} \text { Thres }_{1 n}
\end{aligned}
$$

The deriving of weights will be analyzed in our other paper. Here, we only provide the parameter-values of "Lena" in Q1: $C_{1 b}=0.5, C_{1 e}=1.0, C_{2 b}=0.6, C_{2 e}=0.2, C_{3 b}=0.8, C_{3 e}=0.1$.

\subsection{Adaptive Thresholding in Contourlet Domain}

The soft thresholding is adopted in our algorithm. It shrinks the contourlet coefficients above and below the threshold, and reduces them toward zero.

$$
\text { if } \begin{aligned}
B M(i, j) & =1 \\
V(i, j) & =\left\{\begin{aligned}
V(i, j)-\text { Thres }_{b p} & \text { for } V(i, j) \geq \text { Thres }_{b p} \\
V(i, j)-\text { Thres }_{b n} & \text { for } V(i, j) \leq \text { Thres }_{b n}
\end{aligned}\right.
\end{aligned}
$$

\section{EXPERIMENTAL RESULTS}

In order to validate the high efficiency of the proposed deblocking algorithm, extensive experiments have been carried out on two $512 \times 512$ standard test images "Lena", and "Barbara". Two JPEG quantization tables in [8] are used to compress images. "Lena" contains mainly smooth regions while "Barbara" contains a large area of texture. Fig. 4 and Fig. 5 shows, respectively, the original "Lena" and "Barbara", BDCT compressed "Lena" and "Barbara" (using Q3) with BA, and the deblocked "Lena" and "Barbara" using the proposed algorithm. The BDCT compressed "Lena" and "Barbara" in figures have peak signal to noise ratio (PSNR) of $27.38 \mathrm{~dB}$ and $24.03 \mathrm{~dB}$ respectively, whereas the deblocked images have PSNR of $28.76 \mathrm{~dB}$ and $24.73 \mathrm{~dB}$ respectively. It is clear that the better subjective quality can be acquired by the proposed deblocking algorithm when comparing the cropped high-density edge and texture areas of the images shown in Figs. 4 and 5.

We compare the performance of our algorithm with Hsung's wavelet singularity detection (WSDH) algorithm [2], MPEG-4 VM postfiltering (MPG4) algorithm [4], Yang's spatially adaptive POCS (POCSY) algorithm [5], Paek's POCS (POCSP) algorithm [6], Xiong's overcomplete wavelet deblocking (WDX) algorithm [7] and Liew's overcomplete wavelet deblocking (WDL) algorithm [8]. Here, POCSY and POCSP are iterative while the other algorithms are noniterative. The PSNR results for the different algorithms are shown in Table 1. It is evident that our algorithm consistently outperforms WSDH, MPG4, POCSY, WDX and WDL in terms of PSNR. Our algorithm outperforms POCSP for "Lena" and "Barbara" in Q3, only is inferior for "Barbara" in Q1.

Table 1. Comparison of deblocking results on two images (average PSNR in $\mathrm{dB}$ ).

\begin{tabular}{c|c|c|c|c} 
& \multicolumn{2}{|c|}{ Lena } & \multicolumn{2}{c}{ Barbara } \\
\hline Quantizer & Q1 & Q3 & Q1 & Q3 \\
\hline Test image & 30.70 & 27.38 & 25.94 & 24.03 \\
\hline WSD $_{H}$ & 31.30 & 27.89 & 24.65 & 23.63 \\
\hline MPG4 & 31.21 & 28.10 & 26.09 & 24.37 \\
\hline POCS $_{\mathrm{Y}}$ & 31.31 & 28.29 & 26.40 & 24.45 \\
\hline POCS $_{\mathrm{P}}$ & 31.63 & 28.51 & $\mathbf{2 6 . 6 4}$ & $\mathbf{2 4 . 7 3}$ \\
\hline WD $_{\mathrm{X}}$ & 31.22 & 28.32 & 25.23 & 24.10 \\
\hline WD $_{\mathrm{L}}$ & 31.61 & 28.65 & 26.37 & 24.66 \\
\hline Our method & $\mathbf{3 1 . 6 4}$ & $\mathbf{2 8 . 7 6}$ & 26.47 & $\mathbf{2 4 . 7 3}$
\end{tabular}

\section{CONCLUSION}

In this paper, we proposed a contourlet-based deblocking algorithm. With ss-edge detector, BM and EM of the compressed image are extracted in the same time. The compressed image is filtered in contourlet domain with adaptive thresholds that are obtained based on BM. Comparative study has illustrated that our deblocking 
algorithm has the better visual quality as well as the higher PSNR.

\section{ACKNOWLEDGEMENT}

The authors acknowledge the financial support of RFDP No.20040248047 and NSF No.60502033.

\section{REFERENCES}

[1] S. Liu and A. Bovik, "Efficient DCT-Domain blind measurement and reduction of blocking artifacts," IEEE Trans. Circuits Syst. Video Technol., vol. 12, pp. 1139-1149, Dec. 2002.

[2] T. C. Hsung and D. P. K. Lun, "Application of singularity detection for the deblocking of JPEG decoded images," IEEE Trans. Circuits Syst. II, vol. 45, pp. 640-644, May 1998.

[3] S. Wu, H. Yan, and Z. Tan, "An efficient wavelet based deblocking algorithm for highly compressed images," IEEE Trans. Circuits Syst. Video Technol., vol. 11, pp. 1193-1198, Nov. 2001.

[4] MPEG 4 Verification Model, VM 14.2, pp. 260-264, 1999.

[5] Y. Yang and N. P. Galatsanos, "Projection-based spatially adaptive reconstruction of block-transform compressed images," IEEE Trans. Image Processing, vol. 4, pp. 896-908, July 1995.

[6] H. Paek, R. Kim, and S. Lee, "On the POCS-based postprocessing techniques to reduce the blocking artifacts in transform coded images," IEEE Trans. Circuits Syst. Video Technol., vol. 8, pp. 358-367, June 1998.
[7] Z. Xiong, M. T. Orchard and Y. Zhang, "A deblocking algorithm for JPEG compressed images using overcomplete wavelet representation," IEEE Trans. Circuits Syst. Video Technol., vol. 7, pp. 433-437, Apr. 1997.

[8] A. Liew and H. Yan, "Blocking artifacts suppression in blockcoded images using overcomplete wavelet representation," IEEE Trans. Circuits Syst. Video Technol., vol.14, pp. 450-461, Apr. 2004.

[9] M. Do and M. Vetterli, "Contourlets: A Directional Multiresolution Image Representation," The IEEE International Conference on Image Processing (ICIP'2002), vol. 1, pp. 357-360, 2002 .

[10] M. Do and M. Vetterli, "The Contourlet Transform: An Efficient Directional Multiresolution Image Representation," IEEE Trans. Image Processing, vol. 14, pp. 2091-2106, Dec. 2005.

[11] A. Cunha, J. Zhou and M. Do, "Nonsubsampled Contourlet Transform: Filter Design and Applications in Denoising," The IEEE International Conference on Image Processing (ICIP'2005), vol. 1, pp. 749-752, 2005.

[12] R. Eslami, H. Radha, "Image denoising using translationinvariant contourlet transform," IEEE International Conference on Acoustics, Speech, and Signal Processing (ICASSP'2005), vol. 4, pp. 557-560, 2005.

[13] M. Dow, "Scale Space Edges," http://bdl.uoregon.edu/mri/mark/ss_edges/.

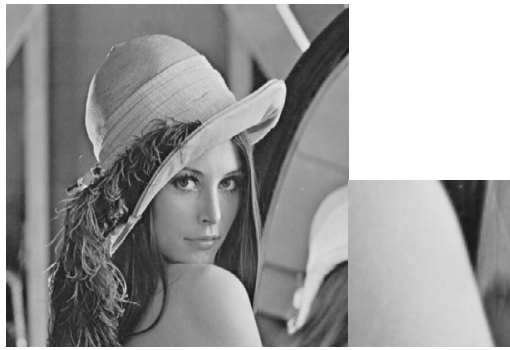

(a)

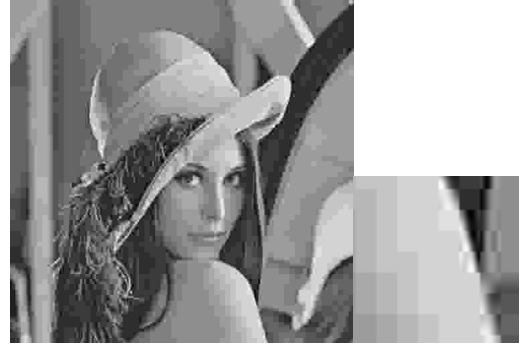

(b)

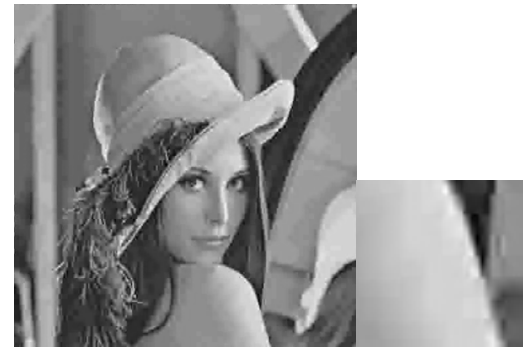

(c)

Fig. 4. Deblocking results using the proposed algorithm for "Lena" (a) original image (b) BDCT compressed image (c) deblocked image.

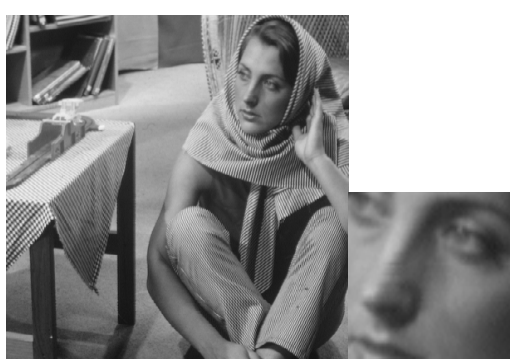

(a)

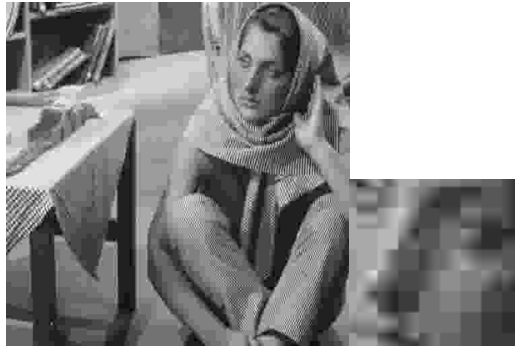

(b)

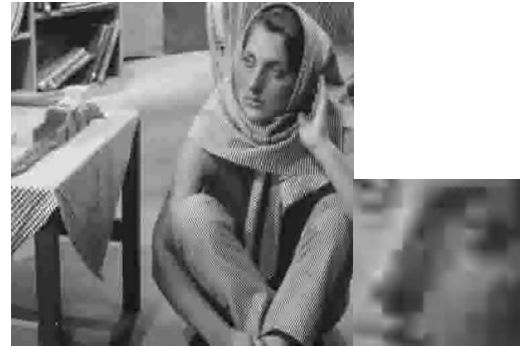

(c)

Fig. 5. Deblocking results using the proposed algorithm for "Barbara" (a) original image (b) BDCT compressed image (c) deblocked image. 